\title{
Algunas notas acerca de la evolución de la Arqueología en Marruecos (1956-2016)
}

\section{Some notes about the evolution of Archaeology in Morocco (1956-2016)}

\author{
Enrique Gozalbes Cravioto ${ }^{1}$
}

Recibido: 19-08-2017

Aceptado: 19-09-2018

\section{Resumen}

En el trabajo analizamos la evolución experimentada por la arqueología marroquí en los sesenta años transcurridos desde el acceso a la independencia por parte de este país del Magreb. El análisis se articula en torno a tres partes: el primer periodo de la evolución de la arqueología (1956-1990), el segundo periodo caracterizado por una mayor presencia de arqueólogos marroquíes, y con especial dedicación a la arqueología árabe-islámica, y finalmente se realiza un análisis de la revista Bulletin d'Archéologie Marocaine, la más importante sobre la arqueología de Marruecos.

Palabras clave: historiografía, arqueología, estudios pos-coloniales, prehistoria, mundo romano, arqueología árabeislámica.

\begin{abstract}
In the paper we analyze the evolution experienced by Moroccan archeology in the sixty years since the country's access to independence from the Maghreb. The analysis is structured around three parts: the first period of the evolution of archeology (1956-1990), the second period characterized by a greater presence of Moroccan archaeologists, and with special dedication to Arab-Islamic archeology, and finally we realized an analysis of the journal Bulletin d'Archéologie Marocaine, the most important magazine in the archeology of Morocco.
\end{abstract}

Keywords: historiography, archeology, post-colonial studies, prehistory, Roman world, Arab-Islamic archeology.

\section{INTRODUCCIÓN}

En los últimos años, y sobre todo en el ámbito español, se ha comenzado a prestar una atención especial a la Historia de la Arqueología en Marruecos. Este hecho está ligado de forma bastante estrecha a la voluntad de recuperar la memoria perdida de la olvidada acción arqueológica desarrollada por parte de los investigadores españoles (C. L. Montalbán, P. Quintero, C. Morán, M. Tarradell) en la época del
Protectorado en el Norte de Marruecos (1912-1956), cuestiones acerca de las que ya existe una abundante biografía (entre otros Gozalbes, 2012a; Parodi, 2017). Por el contrario, mucho menos se ha estudiado en relación a la revisión historiográfica de la acción arqueológica protagonizada por los franceses en la misma época, en especial por parte de L. Chatelain o de R. Thouvenot, quienes desde su dirección de la arqueología marroquí (en 1914-1938 y 1938-1955 sucesiva-

1 Universidad de Castilla-La Mancha,

Enrique.Gozalbes@uclm.es 
mente) sentaron las bases del desarrollo de la arqueología marroquí con posterioridad. Pero aún así, incluso hasta el momento no se ha realizado ningún análisis acerca de la evolución de la acción y de la investigación arqueológica en el vecino país norteafricano después de que el mismo recuperara la independencia, ya como país moderno, a partir de 1956.

La presente contribución tiene como objetivos el señalar las principales líneas evolutivas que ha seguido la arqueología marroquí después de su constitución como país independiente moderno, cuestiones bastante desconocidas incluso en los países que como Francia, España o Italia mantienen un relevante o cierto nivel de colaboración. De igual forma, pretendemos señalar el momento y las circunstancias del cambio producido hacia el modelo actual que supone el protagonismo de los propios marroquíes en la arqueología de su país. Finalmente, como un documento significativo para acreditar aspectos que serán señalados, planteamos un análisis de la evolución del principal órgano de difusión de la arqueología de Marruecos: el Bulletin d'Archéologie Marocaine. En cualquier caso, en el análisis prestaremos una especial atención al tránsito de una arqueología practicada exclusivamente por extranjeros, típicamente de un medio colonial, a una investigación arqueológica compartida, con fuerte protagonismo de miembros de otras nacionalidades, pero en la que los marroquíes ya tienen un activo campo de presencia, con sus potencialidades y también con sus fuertes limitaciones.

\section{LAS PRIMERAS ETAPAS (1956-1990)}

En un principio, después del acceso a la independencia del país en 1956, los cambios efectuados en la arqueología marroquí fueron mínimos, en una transición que significó prácticamente el mantenimiento de las mismas estructuras pre-existentes a la propia independencia. En este sentido el Service des Antiquités du Maroc, establecido en la zona francesa de Marruecos desde la época de L. Chatelain, simplemente absorbió la Inspección de Antigüedades de la zona de Protectorado español en el Norte del país (con el abandono subsiguiente de los estudios realizados en la zona de Tetuán por parte de M. Tarradell). El Servicio de Antigüedades, a partir del año 1956, desde la misma estructura anterior (Euzennat, 1962) contaría con la figura de un Director representada por M. Euzennat, cuya investigación fundamental se dirigió al estudio de los campamentos militares en Marruecos, y un SubDirector dedicado a las antigüedades prehistóricas, al cargo de las cuáles estuvo G. Souville, principalmente investigador del material en los Museos y especialista en la época post-paleolítica. Se mantendría en los años subsiguientes una importante producción editorial por parte del Servicio de Antigüedades, pues la serie Publications du Service des Antiquités du Maroc, hasta ese momento una revista, pasaría a ser monográfica, y desde el año 1956 contaría con una nueva revista: el Bulletin d'Archéologie Marocaine.

Así pues, esta estructura tradicional establecida por los franceses se mantuvo, potenciando en esos momentos las investigaciones de personas de esa nacionalidad que se había estado desarrollando desde unos momentos anteriores, en especial sobre el Paleolítico Inferior (P. Biberson), las excavaciones prehistóricas en la gruta de Taforalt (J. Roche), el estudio y catalogación de los grabados rupestres del Atlas (J. Malhomme), o los estudios en Tánger y las excavaciones en Lixus (M. Ponsich). A nivel internacional, la arqueología marroquí aspiró a organizar el V Congreso Panafricano de Prehistoria, e incluso se llegó a aprobar que el mismo se celebrara de forma conjunta entre España (islas Canarias) y Marruecos, pero finalmente éste país retiró su compromiso por lo que el mismo tuvo como centro tan sólo las Canarias (Diego Cuscoy ed., 1965; Gozalbes, 2006). Esta retirada evitó a Marruecos el organizar un evento científico internacional del que ha estado privado hasta el momento como una de las fuertes limitaciones de la arqueología del país magrebí; debe destacarse sin embargo que el próximo Congreso Panafricano de Arquología y Prehistoria, en su XV edición, se celebrará en Rabat en 2018 (55 años después).

El primer cambio en la arqueología marroquí se produjo en 1963-1964 con la sustitución de la dirección anterior que participó, desde entonces, en el repliegue de los investigadores franceses del Magreb, en especial hacia la Universidad de Aix-en-Provence, en donde se realizaría en 1967 la fundación de la revista Antiquités Africaines (dedicada al conjunto del Magreb). Según el propio testimonio personal de sus protagonistas (G. Souville, testimonio oral) correspondió a un cambio personal bastante abrupto en especial en relación con M. Euzennat, en particularmente malas relaciones con una buena parte de los restantes investigadores franceses relacionados con Marruecos. Pese a todo, es cierto que no se producirían cambios conceptuales relevantes en los siguientes años, en los que se mantuvo la actuación omnipresente de muchos investigadores franceses en el país. En general también se mantuvo la separación conceptual bastante potente en la época del Protectorado entre los que significaba la arqueología prehistórica y sobre todo clásica, por un lado, y los estudios sobre el registro material árabe-islámico clasificados en el ámbito de las Bellas Artes, por el otro (una interpretación diferente, más optimista, en Naji, 2011). El predominio de la acción francesa condujo a que se mantuviera bien evidente la atención de los arqueólogos sobre el mundo clásico, mientras por el contrario disminuyó notablemente la dedicación a la prehistoria que quedó especialmente en el desarrollo de los estudios por parte del investigador francés 
Georges Souville ya emigrado a Francia (Gozalbes, 2012a; 2012b).

En cualquier caso, fue una época de escasísimo interés social y político por la arqueología en el país marroquí, actividad que se relacionaba más bien con el interés francés o "colonial", aunque pudiera tener su atractivo turístico, todavía no del todo bien desarrollado en esos momentos. Además, según Francia iba dejando la tutela sobre Marruecos en el terreno económico, se iba acentuando una época de escasísimas inversiones en arqueología, congruente con la escasa atención socio-política. Así la actividad efectiva en los campos arqueológicos se fueron cerrando progresivamente, por ejemplo Tamuda tuvo su última campaña de limpieza en el año 1964, en Lixus se suspendieron las excavaciones en 1967 en un paréntesis que duraría tres décadas, y tan sólo se mantuvieron actividades muy limitadas en Volubilis o Banasa, quizás algo más importantes en Sala (Rabat) desarrolladas por J. Boube. Aparte de los escasos fondos dedicados a una actividad que se ligaba erróneamente por muchos a los intereses "colonialistas", también influía mucho la práctica ausencia de marroquíes especialistas en Arqueología, patrimonio o Historia Antigua.

Pese a todo se establecerán algunos equipos de investigación básicamente por parte de franceses o de otros extranjeros, aunque es cierto que en algunos de ellos se buscaría una colaboración marroquí. Así, con el tiempo, la Mission préhistorique et paléontologique française au Maroc (A. Debenath y J. P. Daugas), aunque es cierto que con publicaciones muy posteriores, sobre todo las excavaciones desarrolladas en Dchar Jdid, la antigua colonia romana de Iulia Constantia Zilil (dirigidas por E. Lenoir), pero también (y ello es muy significativo) ahora se prestará una atención ya más especial al campo de la arqueología islámica, como en el caso de las excavaciones en Belyounech al Oeste de Ceuta (C. Terrasse y Joudia HassarBenslimane), o en diversos emplazamientos (tales como Nakur) en el Rif (P. Cressier), o también las realizadas por un equipo arqueológico norteamericano en el centro de Qsar Saghir que llegaba incluso al estudio de la fortaleza portuguesa (C. Redman).

En lo que respecta a esta época podemos destacar el hecho de que después de una ausencia de muchos años, en 1987 se presentaron las primeras tesis doctorales sobre la Mauretania Tingitana en Universidades españolas, F. López Pardo (1987) en la Universidad Complutense y E. Gozalbes Cravioto (1987) en la de Granada, que marcarían de una o de otra forma la senda para las realizadas por otros españoles ya en fases posteriores como D. Bernal (1997), N. Villaverde (2000), Ll. Pons (2003)... Sin embargo, es cierto que la formación y tradición francesa tuvo mucho más peso en el terreno de la arqueología y del mundo antiguo en Marruecos. Así hemos realizado un breve análisis a partir de los datos recogidos, sobre dos décadas (1972-
1992) de Tesis Doctorales en las Universidades francesas, teniendo en cuenta las Tesis de Tercer Ciclo, de Estado y Nueva Tesis. En esos años, hemos computado un total de 39 tesis referidas a Marruecos, desde la Prehistoria al patrimonio árabe-islámico medieval y moderno (Benhada y Lmoubariki, 1994).

En estas tesis tan sólo hay dos referidas a la arqueología más primitiva, en ambos casos elaboradas por doctorandos marroquíes, en 1985 la de M. A. ElHajraoui sobre las industrias prehistóricas de la zona de La Mamora, y la de 1991 de Y. Bokbot sobre los monumentos funerarios en el Marruecos protohistórico. Es cierto que la lista que se recoge no es completa por cuanto en la misma no aparecen reflejadas las tesis de G. Souville de 1972, dedicada al Atlas prehistórico de Marruecos, y de A. Salih, del año 1990, sobre el Musteriense y el Ateriense marroquíes. Respecto a la antigüedad clásica se enumeran un total de 22 tesis, de ellas 7 elaboradas por doctorandos franceses (las más antiguas en 1972 la de M. Ponsich, la de Ph. Schmitt en 1973, la de 1976 de J. Desanges y la de 1982 de A. Jodin) y 15 presentadas por marroquíes (las más antiguas de Mustapha Moulay Rachid en 1981 sobre la Mauretania y sus relaciones con el imperio romano, la de Halima Ghazi Ben Maissa sobre el culto imperial en 1982, la de A. Akerraz en 1985 sobre el Sur de Marruecos desde Diocleciano a los Idrisíes, y la tesis de H. Limane en 1988 sobre las cerámicas sigillatas romanas en la Tingitana). Por tanto, como puede verse dichas tesis doctorales sobre arqueología elaboradas por investigadores marroquíes comenzaron a presentarse a partir de los años ochenta.

Y respecto a la arqueología o patrimonio árabeislámico, la mayoría de las tesis fueron ya efectuadas por marroquíes, aunque las más antiguas fueron de los franceses M. Terrasse (arqueología y arte de época de los Merinidas) en 1979, y de P. Cressier (arqueología del Rif) en 1981, abriendo nuevamente el camino transitado por la arqueología del país magrebí. El conjunto refleja un inicio de la dedicación de los marroquíes a estos temas prácticamente avanzados los años ochenta, con un debut con la tesis de Joudia Hassar Benslimane sobre Recherches archéologiques à Salé en el año 1979, en general con una dedicación similar a la antigüedad y a la arqueología y al patrimonio árabe-islámicos.

\section{LA CREACIÓN DEL INSAP Y LA COOPERACIÓN INTERNACIONAL}

La evolución política del Reino de Marruecos evidenció la fuerte limitación de la organización de la arqueología, así como los resultados relativamente pobres de la misma a partir de mediados de los años setenta. Había que responder al reto de una modesta pero creciente atención de un sector de la juventud 
marroquí hacia la arqueología y el patrimonio cultural, incluido en ello la propia museística (más amplia que la arqueología). Por otra parte, quedaba claro para el país magrebí que tenía que aumentar sustancialmente el presupuesto dedicado a la arqueología y al patrimonio, pero que al final de cuentas los recursos a emplear siempre serían muy limitados en relación a las necesidades. En este sentido en 1985 se creó el INSAP (Institut National des Sciences de l'Archéologie et du Patrimoine), que expresamente se constituía como un órgano superior de enseñanza y de formación para la investigación en las ciencias de la arqueología y el patrimonio. Y en segundo lugar, a través sobre todo del propio INSAP, se trataba de fomentar la cooperación internacional, con la firma de convenios con equipos de investigación (con sus presupuestos) extranjeros, en los que se integrarían estudiosos marroquíes para desarrollar trabajos en el país.

La formación de un grupo reducido pero activo de arqueólogos marroquíes se plasmó sobre todo ya en los años noventa. En este sentido, si tenemos en cuenta la completa bibliografía sobre el Marruecos antiguo elaborada por Brouquier-Reddé y Lenoir (2000) podemos observar que, excepto algunos trabajos de A. Akerraz, las primeras publicaciones de arqueólogos marroquíes (Y. Bokbot, F. Z. El Harif, A. El Khayari, M. Kbiri Alaoui, H. Limane o A. Ichkakh) y de estudiosos de la antigüedad (Ghazi be Maisa, C. Hamdoune, M. Madjoub....) tan solo comenzaron a producirse en los años noventa. Y también ya en la época del cambio de siglo cuando algunos investigadores marroquíes comenzaron a participar en los Coloquios del África Romana organizados por la Universidad italiana de Sassari.

También las principales actividades de cooperación internacional se comenzarán a implementar sobre todo a partir de mediados de la última década del siglo XX. Es cierto que siempre los franceses mantendrán un protagonismo mayor al respecto, fundamentados sobre todo en las actuaciones de M. Lenoir (y E. Lenoir) y R. Rebuffat, siguiendo la tradición de la presencia arqueológica francesa en el exterior (Bazzana y Boccum, 2004; Varios Autores 2005), que amén de su potencia en sí misma, incluso en la multiplicación de misiones arqueológicas, ha buscado en el modelo del partenariado una revisión y actualización de sus planteamientos (Kasbarian, 2012).

Como señalamos resaltan las actuaciones de $\mathrm{M}$. Lenoir y de R. Rebuffat, destacando en primer lugar el desarrollo del registro arqueológico en la colonia romana de Zilil (Lenoir 2005) y también la "Mission de prospections du bassin du Sebou", que se incardinó en el proyecto marroquí dirigido por A. Akerraz de elaboración de una nueva Carta arqueológica (Rebuffat, 2011). Otros proyectos de colaboración importantes han sido los referidos al estudio de los templos romanos en la Tingitana, la nueva investiga- ción y revisión acerca de los hornos alfareros de Kouass desarrollado por M. Kbiri Alaoui y V. Bridoux (Kbiri Alaoui 2007; Bridoux y Kbiri Alaoui 2012), el estudio de los monumentos religiosos en especial en Lixus por parte de V. Brouquier-Reddé, A. El Khayari y A. Ichkhakh (2001), así como especialmente en lo últimos años las investigaciones arqueológicas realizadas en Rirha y que han dado lugar a la publicación de varios volúmenes (Callegarin, Kbiri Alaoui, Ichkhakh y Roux eds., 2016-17).

Igualmente, desde esa época, y sobre todo a partir de la iniciativa de M. Fernández Miranda, se potenciará la participación de algunos arqueólogos españoles en la puesta en marcha de estas investigaciones hispano-marroquíes (Onrubia, 1996). Así podemos destacar algunas investigaciones desarrolladas a partir de ese momento (Gozalbes, 2012c 349-350), como la participación de F. López Pardo en los estudios sobre la cerámica y otros elementos fenicios de Mogador (López Pardo y Mederos, 2008; López Pardo et alii, 2011), los trabajos de J. Onrubia de prospección en la zona del Oued Laou en el Norte de Marruecos, así como la prospección de la prehistoria tardía en la zona de Tarfaya (Onrubia, 2004) y el SusTekna (Onrubia et alii, 2015), sobre todo el desarrollo de excavaciones arqueológicas durante muchos años en la ciudad fenicia, mauritana y romana de Lixus (Aranegui, 2004; Aranegui, 2017), que ha dado lugar a la publicación de varios volúmenes sobre la ciudad (Aranegui, 1999-2010), los proyectos de estudio del Museo Arqueológico de Tetuán (Ramos, Bernal, Zouak y Raissouni 2007), las excavaciones desarrolladas en Tamuda desarrolladas por parte de las Universidades de Cádiz, más centrada en los vestigios de la ciudad mauritana (Bernal, Raissouni, Verdugo y Zouak, 2013) y de Huelva (vid. especialmente de este último equipo las publicaciones con nuevos y relevantes datos sobre la evolución del castellum romano, Campos y Bermejo 2013; Campos y Bermejo 2015), así como la elaboración de la carta arqueológica del Norte de Marruecos, trabajo amplio de prospección de la zona oriental de la península Noroeste de Marruecos, y que ha sido con razón calificado de "proyecto ilusionante para el desarrollo de la puesta en valor del patrimonio histórico y arqueológico" (Bernal, El-Khayari, Raissouni, Ramos y Zouak, 2008).

De forma complementaria con los trabajos de cooperación desarrollados por marroquíes con franceses y españoles, también podemos destacar otras intervenciones. En arqueología prehistórica el desarrollo del proyecto franco-marroquí de revisión de la prehistoria marroquí, con especial incidencia en el Paleolítico Inferior, desarrollado por J. P. Daugas y colaboradores, la prehistoria de Tánger desarrollada por un equipo belgo-marroquí dirigido por M. Otte (Otte y Bouzougar 2004) y la excavación y estudio de la presencia fenicia 
en Mogador, realizada junto con el Instituto Arqueológico Alemán bajo la coordinación de Dirce Marzoli (Marzoli y El Khayari, 2010). Podemos también destacar los estudios dirigidos por el investigador británico Nick Barton en Taforalt, en Dar es Soltan (Barton, Bouzougar et alii, 2009) y últimamente en Gar Cahal (resultados sin publicar en este caso). También destacamos el proyecto de investigación italo-marroquí en el Rif y que ha dado lugar a la localización de un nuevo emplazamiento con restos fenicios en Cabo de Agua, al Este de Melilla (Vismara, 2003).

Finalmente, uno de los aspectos que puede ayudar a reflejar una parte de la arqueología marroquí es la formación de los "laureados" a partir de 1990 por parte del propio INSAP, que marcan la preparación de los futuros especialistas. La información disponible al respecto es bastante atrasada y corresponde a la obtenida de la página web oficial de esta institución. En este sentido, el número de "laureados" hasta el año 2008 no era demasiado elevado, pero el reflejo de los mismos por especialidades refleja la realidad de la atención por parte de las modernas generaciones marroquíes. El mayor número de laureados está representado por la arqueología islámica (el $22^{\prime} 7 \%$ ), seguida por la sección estrictamente de monumentos históricos o patrimonio (el 20`7 \%), en tercer lugar por la propia museología (el 16’2\%), en cuarto lugar por la prehistoria (el 15’3 \%), y tan sólo en quinto lugar toda la "arqueología pre-islámica" (con el 13’3\%), sólo por encima de la AntropologíaEtnografía (con el 11'8\%). Estas cifras señalan la dificultad de extensión de la arqueología clásica, frente a otras ramas cercanas como la arqueología islámica, el patrimonio o la museología, que entre todas representan el $60 \%$ de los "laureados" en la última década del siglo $\mathrm{XX}$, la única de la que disponemos de datos. Desde entonces no parece que la atención por la arqueología clásica haya aumentado sino más bien todo lo contrario, lo que confirma otros datos que iremos recogiendo.

\section{El Bulletin d’Archéologie Marocaine}

La revista Bulletin d'Archéologie Marocaine apareció por vez primera en el año 1956, y hasta 2016 ha publicado un total de 22 números (aunque con un problema evidente de numeración puesto que es el número 23 oficialmente). Este hecho irregular parte de que el número 13 (entre el de 1979-1980 y el de 1981-1982) simplemente no existe, lo que no deja de constituir un hecho especialmente singular. Ello significa la publicación de un número de la revista cada 2`7 años. En la última década del siglo, la aparición de un solo número (en 1998 distante diez años del anterior) señalaba que la publicación prácticamente había desaparecido, lo que parece indicar que llevó particularmente mal su asunción por parte del INSAP en esos momentos. Sin duda, en esa casi extinción precisamente influyó la falta de investigaciones completas sobre la antigüedad que hasta ese momento, como luego veremos, constituían el cuerpo principal de la revista.

En una revista en la que hay que valorar sobre todo su capacidad relativa de continuidad durante seis décadas, hecho prácticamente singular en el Magreb, no es nuestro objetivo el entrar a exponer los principales problemas de edición, presentación, o maquetación, que pueden detectarse en el pasado lejano y cercano y en el presente, que por otra parte parecen muy evidentes ante la mirada europea. En cualquier caso, en una era de "indexación" con criterios e índices de calidad nos parece imprescindible en un análisis acerca de la misma mencionar datos problemáticos como son la extraordinaria irregularidad en la publicación, los grandes problemas tipográficos presentes y los cambios en la misma (frente a la gran calidad de los siete primeros números), así como los potentes problemas en la difusión de la misma en relación con las bibliotecas europeas y norteamericanas (resulta muy difícil su consulta).

Los cuatro primeros números del BAM (1956, 1957, 1958-59 y 1960) estuvieron protagonizados prácticamente sólo por los investigadores franceses presentes en esos momentos en Marruecos, y correspondieron a una alternancia entre la arqueología prehistórica (números 1 y 3) y la clásica (números 2 y 4) bajo la dirección de G. Souville y de M. Euzennat. Prescindimos de ellos en el análisis constatando simplemente que esta primera fase no constituyó sino una continuación de la potente arqueología del final de la época del Protectorado francés. A partir del número 5 de 1964 y hasta el número 8 los cambios en el Servicio de Antigüedades no afectaron a la estructura de la revista que contaría con un noticiario de crónica de la arqueología marroquí (que tan sólo se retomará excepcionalmente en el número 10 de 1976), aunque en este caso ya parece significativo de la voluntad de "marroquinización" que estaba firmada por parte de los dirigentes marroquíes de la arqueología (N. Khatib y M. Bekkari sucesivamente y J. Hassar Benslimane en 1976).

En cuanto a la estructura del BAM también ha sido corriente la división entre los artículos más largos y las notas y documentos, así como en ocasiones en los primeros números entre la prehistoria y la arqueología clásica. Sin embargo, a partir del número 10 de 1976 se establecerá la división más duradera en la estructura entre Prehistoria, Arqueología clásica y Arqueología islámica, en ocasiones con una cuarta sección de Etnología o de Antropología. En lo referido al reparto de los trabajos por especialidades podemos establecer el siguiente cuadro general: 
\begin{tabular}{|l|l|l|l|l|l|l|} 
Datos & $1961-1970$ & $1971-1980$ & $1981-1989$ & $1990-1999$ & $2001-2009$ & $2011-2016$
\end{tabular}

\begin{tabular}{|l|c|c|c|c|c|c|}
\hline Números & 4 & 4 & 4 & 1 & 3 & 2 \\
\hline Prehistoria & 24 & 16 & 16 & 8 & 19 & 12 \\
\hline Clásica & 68 & 21 & 23 & 11 & 21 & 13 \\
\hline Islámica & 2 & 9 & 13 & 5 & 19 & 16 \\
\hline Etnografía & - & - & - & - & 4 & 6 \\
\hline TOTAL & 94 & 46 & 52 & 24 & 63 & 47 \\
\hline
\end{tabular}

Tabla 1. Temática de los articulos publicados en la revista Bulletin d'Archéologie Marocaine entre 1961 y 2016.

Este cuadro marca la existencia de un total de unos 326 trabajos en estos 18 números lo que supone una media de unos 18 trabajos por número (entre artículos, notas y documentos). De ellos la gran mayoría está constituida por la "arqueología pre-islámica" con 157 trabajos lo que supone el $48 \%$ del total. En segundo lugar se encuentra la prehistoria con 95 artículos con un $29 \%$, mientras la arqueología islámica con 64 trabajos, el $19 \%$. Ahora bien, esta relación marca una larga evolución de algo más de medio siglo, pero una mirada más focalizada marca que entre 1960-1980 la arqueología clásica supuso el 63`6 \% y la islámica apenas el $8 \%$, entre 1981 y 2000 la arqueología clásica pasó del $45 \%$ y la islámica el $24 \%$, mientras que en los últimos cinco números (2001-2016) ya la arqueología islámica ha pasado a ocupar el primer lugar en unas proporciones muy equilibradas: $35 \%$ frente a $34 \%$ de la arqueología clásica y el $31 \%$ de la prehistórica. Así pues, en general se produce un mantenimiento de la arqueología prehistórica, en torno al $30 \%$, mientras de forma progresiva disminuye notablemente la presencia de la arqueología clásica (desde algo más del $60 \%$ al $34 \%$ ) mientras se va produciendo un ascenso muy evidente de la presencia de la arqueología islámica (del $19 \%$ hasta el $35 \%$ ).

En lo que se refiere a los autores de las aportaciones, en los primeros números hasta 1980 participaron los investigadores franceses "clásicos" en su actuación en Marruecos (R. Thouvenot, M. Ponsich, A. Jodin, C. Boube-Piccot, J. Marion, A. Luquet, J. Roche, R. Letan, J. Boube) que fueron disminuyendo según fueron desapareciendo en su presencia personal en la investigación en Marruecos. La participación de los marroquíes en esta época prácticamente quedaba reducida a la mencionada elaboración de las Crónicas de la arqueología marroquí.

A partir del número 12 de 1980 comienzan a aparecer investigadores marroquíes como J. Hassar Benslimane, A. Akerraz, A. Oumlil, A. Touri, A. ElKhayari, M. Habibi, M. Kebiri Alaoui, M. Madjoub, M. Zouak, FA. Oumlil, M. Madjoub, H. Limane, etc) ciertamente de una forma muy modesta al principio, de tal forma que los mismos transitan de en torno al $10 \%$ del total en esa primera época, a un $20 \%$ en los números del 14 al 17, a un 58\% en los números del 18 al 21 y a un $55 \%$ del 21 al 23. Así pues, como puede verse, en la revista se salva la tradicional situación derivada de la arqueología colonial de una arqueología en Marruecos sin la presencia protagonista de marroquíes. En cualquier caso su participación es mayoritaria en la arqueología islámica a la que dedicaron el $36 \%$ de los trabajos, seguida de la arqueología clásica con un $33 \%$ y la prehistoria con un $31 \%$, todo ello desde los años ochenta.

Respecto al número de autores de artículos se produce otra evolución en este caso bastante característico de las publicaciones arqueológicas. Así en los números 5 al 12 el número de los mismos fue de unos 12, mientras del 14 al 27 ascendieron a 18, del número 18 al 21 fueron 27 , y finalmente en los últimos números ha sido de 44 de media por número. Este hecho no deriva realmente del aumento de un número de trabajos por número sino de la costumbre creciente de que la publicación no sea firmada por el director individual de la excavación, como era tradicional, sino por una suma de los componentes del equipo de excavación (en el último número uno de los trabajos aparece firmado por 13 y varios por 6 ó por 7 investigadores).

En suma, el análisis del Bulletin d'Archéologie Marocaine desde 1956 hasta 2016 marca una evolución de la arqueología marroquí a grandes rasgos fuertemente paralela, como era difícil que fuera diferente, de la que podemos establecer a partir del análisis de la evolución de las investigaciones realizadas. En los temas hasta finales del siglo XX existe una clara preponderancia de la arqueología clásica, muy en especial en relación a la época romana a la que se dedican el $72 \%$ de los trabajos de esa sección. Sin embargo la arqueología islámica, que aparece como sección hacia 1980, comenzó con una presencia modesta pero ha ido aumentando paulatinamente hasta convertirse en mayoritaria en los últimos núme- 
ros en detrimento de la arqueología clásica. En general la prehistoria aparece como limitada y dependiente fuertemente de los investigadores extranjeros, la arqueología clásica últimamente ofrece indicios de agotamiento para los propios marroquíes, mientras el futuro parece más expedito para la arqueología árabeislámica.

Paralelamente en el análisis de la revista, en lo que respecta a la nacionalidad de los participantes, en los últimos números de la revista continúa habiendo un número importante de franceses, con presencia minoritaria de otras nacionalidades europeas como particularmente la española, pero el número de investigadores marroquíes ha ido aumentando paulatinamente de una forma significativa, en todas las secciones, pero destacando más su presencia en arqueología islámica. Así pues, estas dos líneas, presencia mayoritaria de marroquíes en su propia arqueología, y mayor presencia de la arqueología árabe-islámica con disminución y hasta agotamiento de la clásica, y es de suponer también de la prehistórica (muy dependiente de la presencia de investigadores extranjeros), marca la evolución presente y el previsible futuro de la arqueología en Marruecos.

\section{Agradecimientos}

El presente trabajo se ha desarrollado parcialmente en el marco de nuestra participación en el Proyecto de I+D+i MINECO-Ministerio de Economía y Competitividad, ref. HAR2012-334033 (vigencia 2013-2016), y actualmente (desde 2017) en el I+D+i: Arqueología e interdisciplinariedad: una investigación arqueológico-histórica sobre las relaciones interdisciplinares en la Historia de la Arqueología española (siglos XIX y XX). HAR2016-80271-P. El estudio realizado en los documentos de los Archivos de los Museos Arqueológico de Tetuán y de La Kasbah de Tánger se ha realizado con los permisos correspondientes del Ministerio de Cultura de Marruecos, concedidos en los años 2013 y 2014, por parte del Director de Patrimonio Cultural del Reino de Marruecos Sr. Abdellah Alaoui.

\section{Bibliografía}

Aranegui, C. (1999-2010): Lixus, 3 vols. Valencia.

Aranegui, C. (2004): "Lixus (Larache, Marruecos)". Bienes Culturales, 3: 171-182.

Aranegui, C. (2017): Lixus. Del mito a la Historia. Barcelona.

Barton. N.; Bouzouggar, A.; Collcutt, S.N.; Schwenninger, J.L. y Clark-Balzan, L. (2009): "OSL dating of the Aterian levels at Grotte de Dar es-Soltan I (Rabat, Morocco) and possible impli- cations for the dispersal of modern Homo sapiens". Quaternary Science Reviews, 28: 1914-1931.

Bazzana, A. y Boccoum, H. (eds.) (2004): Du Nord au Sud du Sahara. Cinquante ans d'archéologie française en Afrique de l'Ouest et au Maghreb: bilan et perspectives (Paris, 2002). Saint-Maur-des-Fossés.

Benhadda, A. y Lmoubariki, M. (1994): "Vigt ans de recherches historiques et archeologiques sur le Maroc dans 1'Université française (1972-1992)". Hespéris-Tamuda, 32: 163-186.

Bernal, D. (1997): Economía y comercio de la Bética mediterránea y del "Círculo del Estrecho" en la Antigüedad Tardía (ss. III-VII d.C.) a través del registro anfórico. Tesis Doctoral. Universidad Autónoma de Madrid.

Bernal, D.; Raissouni, B.; Verdugo, J. y Zouak, M. (eds.) (2013): Tamuda. Cronosecuencia de la ciudad mauritana y del castellum romano. Resultados arqueológicos del Plan de Investigación del PET (2008-2010). Cádiz-Tetuán.

Bernal, D.; El-Khayari, A.; Raissouni, B.; Ramos, J. y Zouak, M. (2008): "La Carta arqueológica del Norte de Marruecos (2008-2012). Un ilusionante proyecto hispano-marroquí de valorización patrimonial". En D. Bernal et alii (eds.): En la orilla africana del Círculo del Estrecho. Historiografía y proyectos actuales, Tetuán-Cádiz: 231-263.

Bridoux, V. y Kbiri Alaoui, M. (2012): "Kouass (Asilah, Maroc)". Chronique des actualités archéologiques de l'École Française de Rome (mis en ligne decembre 2012, consultado el 04/08/2017).

Brouquier-Reddé, V. y Lenoir, L. (2000): "Bibliografphie du Maroc Antique". L'Africa Romana, XIII, Roma: 991-1072.

Brouquier-Reddé, V., El Khayari, A. y Ichkhakh, A. (2001): "Recherches sur les monuments religieux de Maurétanie Tingitane: de Louis Chatelain à la misión Temples". Plus d'un siècle de rercherches archéologiques au Maroc. Rabat: 187-197.

Callegarin, L.; Kbiri Alaoui, M.; Ichkhakh, A. y Roux, J.C. (eds) (2016-17): Rirha: site antique el médiéval du Maroc. 3 vols. Paris.

Campos, J. M. y Bermejo, J. (2013): La arquitectura militar del castellum de Tamuda: los sistemas defensivos. Roma.

Campos, J. M. y Bermejo, J. (2015): El urbanismo militar del castellum de Tamuda: la castramentación interior. Roma.

Diego Cuscoy, L. (ed.) (1965): Actas del V Congreso Panafricano de Prehistoria y de Estudio del Cuaternario. Publicaciones del Museo Arqueológico de Tenerife, 5. Tenerife. 
Euzennat, M. (1962): "Le Service des Antiquités du Maroc". Revue Historique, 228 (1): 129-134.

Gozalbes, E. (1987): Economía de la Mauritania Tingitana (Siglos I a. de C.-II d. de C.). Tesis Doctoral. Universidad de Granada.

Gozalbes, E. (2006): "Sobre el V Congreso Panafricano de Prehistoria (Islas Canarias, 1963)". Eres. Arqueología/Bioantropología, 14: 57-68.

Gozalbes, E. (2012a): "El Prof. Dr. Georges Souville (1927-2012). Una semblanza”. Antiquités Africaines, 46-48: 5-8.

Gozalbes, E. (2012b): "El profesor Georges Souville (1927-2012) y la Prehistoria de Marruecos". Revista Atlántica-Mediterránea de Prehistoria y Arqueología Social, 14: 7-13.

Gozalbes, E. (2012c): Marruecos y el África occidental en la historiografía y arqueología española. Ceuta.

Kasbarian, J. M. (2012): “Du désir de rayonnement de l'archéologie française à l'étranger à l'alliance scientifique avec les pays partenaires". Les nouvelles de l'archéologie, 128: 35-40.

Kbiri Alaoui, M. (2007): Revisando Kuass (Asilah, Marruecos). Talleres cerámicos en un enclave fenicio, púnico y mauritano. Valencia.

Lenoir, E. (2005): "La ville romaine de Zilil du Ier siècle av. J. C. au IV siècle ap. J. C.”. Pallas, 68: 65-76.

López Pardo, F. (1987): Mauritania Tingitana: de mercado colonial púnico a provincia periférica romana. Tesis Doctoral. Universidad Complutense de Madrid.

López Pardo, F. y Mederos, A. (2008): La factoría fenicia de la isla de Mogador y los pueblos del Atlas. Sevilla-Tenerife.

López Pardo, F.; El Khayari, A.; Hassini, H.; Kbiri Alaoui, M.; Mederos, A.; Peña, V.; Suárez Padilla, J.; Carretero, P. y Mlilou, B. (2011): “Prospección arqueológica de la isla de Mogador y su territorio continental inmediato. Campaña de 2000". Canarias Arqueológica (Eres), 19 (1): 109-147.

Marzoli, D. y El Khayari, A. (2010): "Vorericht Mogador (Marokko) 2008”. Madrider Mitteilungen, 51: 61-108.
Naji, S. (2011): "Archéologie coloniale au Maroc 1920-1956: civiliser l'archaïque”. Les Nouvelles de l'Archéologie, 126: 23-28.

Onrubia, J. (1996): “El profesor Manuel FernándezMiranda y la cooperación arqueológica hispanomarroquí". Homenaje al Profesor Manuel Fernández-Miranda. Complutum Extra, 6: 23-27.

Onrubia, J. (2004): "El proyecto de cooperación hispano-marroquí en materia de arqueología y patrimonio. Investigaciones arqueológicas (19981999)". Bienes Culturales 3: 183-192.

Onrubia, J.; Bokbot, Y.; Amani, F.; Cáceres, Y.E.; González Marrero, $\mathrm{M}^{\mathrm{a}}$. del C.; Izquierdo, R.; Ares, J. de J.; Laïtouss, L.; López-Menchero, V.M.; Marchante, A.; Moreno, M. y Rodríguez Santana, C.G. (2015): "Investigaciones arqueológicas en la región del Sus-Tekna (Marruecos): informe preliminar de los resultados de la campaña de 2014". Informes y Trabajos, 12: 315-344.

Otte, M.; Bouzouggar, A. y Kozlowski, J. (2004): La Préhistoire de Tanger (Maroc). Lieja.

Pons Puyol, L. (2003): Organización y función de la Mauretania Tingitana en el Imperio Romano (siglos I-III d.C.). Tesis Doctoral. Universidad de Barcelona.

Ramos, J.; Bernal, D.; Zouak, M. y Raissouni, B. (2007): "Nuevas propuestas de investigación arqueológica en el Norte de Marruecos". Revista Atlántico-Mediterránea de Prehistoria y Arqueología Social, 9: 283-290.

Rebuffat, R. (2011): "La carte archéologique du Maroc". Les Nouvelles de 1'Archéologie, 124: 1620.

Villaverde Vega, N. (2000): Tingitana durante la antigüedad tardía (ss. III-VII d.C.). Autoctonía y romanidad en el extremo occidente mediterráneo. Tesis Doctoral. Universidad Autónoma de Madrid.

VV.AA. (2005): Archéologies. 20 ans de recherches françaises dans le monde. Paris.

Vismara, C. (2003): Ricerche archeologique italomarrocchine nel Rif, 2000-2003. Roma. 